\title{
Stage I Thymic Epithelial Neoplasm AJCC v8
}

National Cancer Institute

\section{Source}

National Cancer Institute. Stage I Thymic Epithelial Neoplasm A/CC v8. NCI Thesaurus. Code C136322.

Stage I includes: T1a, b, N0, M0. T1a: Tumor with no mediastinal pleura involvement. T1b: T umor with direct invasion of mediastinal pleura. N0: No regional lymph node metastasis. M0: No distant metastasis. (AJCC 8th ed.) 